\title{
Laboratory Diagnosis of Severe Acute Respiratory Syndrome- related Coronavirus-2 Infection
}

\author{
Aruna Poojary
}

\begin{abstract}
Severe acute respiratory syndrome-related coronavirus-2 (SARS-CoV-2) is a novel coronavirus responsible for the current pandemic of coronavirus disease-2019 (COVID-19) leading to respiratory tract infections including adult respiratory distress syndrome (ARDS). Microbiology confirmation with a real-time polymerase chain reaction (RT-PCR) is essential for diagnosis of COVID-19 disease. Real-time polymerase chain reaction has an important role to play in individual patient management as well as in part of pandemic control measures like isolation, quarantine, and containment. Serological methods for antibody detection play an important role in population surveillance and do not have much role in the diagnosis of the disease.
\end{abstract}

Keywords: COVID-19, Laboratory diagnosis, Severe acute respiratory syndrome-related coronavirus- 2 .

Pediatric Infectious Disease (2020): 10.5005/jp-journals-10081-1234

\section{INTRODUCTION}

The novel coronavirus disease-2019 (COVID-19) now called as the severe acute respiratory syndrome-related coronavirus-2 (SARSCoV-2) was identified as the causative pathogen of pneumonia in Wuhan city (Hubei province), China, in early December 2019.1,2 The disease caused by the virus is referred to as COVID-19 and the World Health Organization (WHO) declared it as a pandemic on March 11, 2020. The virus has the basic reproducibility number (R0) of 2.2 which means that for every one person infected at least 2.2 people in turn can get infected. ${ }^{3}$ The main route of transmission is droplet spread and when one comes in contact with contaminated fomites on which the droplets fall and remain viable until the droplet dries up. ${ }^{4}$

\section{Importance OF Laboratory Testing IN Coronavirus Disease-2019}

The symptoms of COVID-19 disease are nonspecific and mimic any other respiratory viral illness. Patients present with fever, dry cough, cold, sneezing, and shortness of breath, etc. ${ }^{4}$ As a result laboratory confirmation of SARS-CoV-2 is essential to confirm the disease. Laboratory testing also helps understand how the virus behaves in certain high risk population, e.g., elderly patients, immunosuppressed, diabetes, hypertension, and cardiovascular diseases, etc. ${ }^{4}$ Unless the presence of the virus is confirmed in clinical specimens, it is impossible to understand the dynamics of disease transmission and whether measures undertaken to contain the pandemic are working. Thus, laboratory testing for SARS-CoV-2 is not only important from an individual perspective but also from the societal and global prevention perspective.

\section{Whom to Test?}

It is best to follow local guidelines: as for India, The Indian Council of Medical Research (ICMR) issues the strategy for testing which is revised from time to time.
Department of Pathology and Microbiology, Breach Candy Hospital Trust, Mumbai, Maharashtra, India

Corresponding Author: Aruna Poojary, Department of Pathology and Microbiology, Breach Candy Hospital Trust, Mumbai, Maharashtra, India, Phone: +91 9820109602, e-mail: arunapoojary@gmail.com

How to cite this article: Poojary A. Laboratory Diagnosis of Severe Acute Respiratory Syndrome-related Coronavirus-2 Infection. Pediatr Inf Dis 2020;2(2):70-74.

Source of support: Nil

Conflict of interest: None

Strategy for Coronavirus Disease-2019 Testing in India (Version 5, dated $18 / 05 / 2020)^{5}$

1. All symptomatic (ILI symptoms) individuals with history of international travel in the last 14 days.

2. All symptomatic (ILI symptoms) contacts of laboratory confirmed cases.

3. All symptomatic (ILI symptoms) health care workers / frontline workers involved in containment and mitigation of COVID19.

4. All patients of Severe Acute Respiratory Infection (SARI).

5. Asymptomatic direct and high-risk contacts of a confirmed case to be tested once between day 5 and day 10 of coming into contact.

6. All symptomatic ILI within hotspots/containment zones.

7. All hospitalised patients who develop ILI symptoms.

8. All symptomatic ILI among returnees and migrants within 7 days of illness.

9. No emergency procedure (including deliveries) should be delayed for lack of test. However, sample can be sent for testing if indicated as above (1-8), simultaneously.

Testing Newborns of Coronavirus Disease Suspect or Positive Mothers ${ }^{6,7}$

No specific mention has been made in the above ICMR strategy for newborns of COVID suspect or positive mothers. The American

(c) The Author(s). 2020 Open Access This article is distributed under the terms of the Creative Commons Attribution 4.0 International License (https://creativecommons. org/licenses/by-nc/4.0/), which permits unrestricted use, distribution, and non-commercial reproduction in any medium, provided you give appropriate credit to the original author(s) and the source, provide a link to the Creative Commons license, and indicate if changes were made. The Creative Commons Public Domain Dedication waiver (http://creativecommons.org/publicdomain/zero/1.0/) applies to the data made available in this article, unless otherwise stated. 
Academy of Pediatrics has however issued an initial guidance for newborns. The rationale for neonatal screening as described is as follows:

- Testing must be done if facilities are available, as it would help facilitate plan of care after hospital discharge.

- If testing is not readily available and/or in short supply clinical monitoring may be undertaken.

- Testing infants who may require prolonged intensive care will help optimize the use of infections control precautions and PPE use especially if the result is negative.

\section{When to Test?}

The optimal time to test is important to identify patients who can transmit the disease. This helps in strengthening prevention strategies. While more studies are needed to understand the transmission dynamics, $\mathrm{He} X$ et al. Wölfel $\mathrm{R}$ et al. point out that viral shedding begins $2-3$ days before the first symptoms appear and continues up to 8 days after symptom onset beyond which the virus has no longer been cultured. ${ }^{8,9}$

Hence, the optimal time to test for COVID-19 as per ICMR is as follows:

- Symptomatic patients soon after symptom onset to up to 8 days.

- Asymptomatic high-risk contacts should be tested between 5 days and 14 days after exposure, preferably later than earlier to optimize the testing strategy.

Local state guidance must be followed which may be updated from time to time based on the epidemiology of the disease in that state.

\section{Optimal Time for Testing Newborns ${ }^{6,7}$}

While the optimal time for testing a newborn is not yet known, the aim of selecting an optimal time is to be able to distinguish between transient viral colonization and established infection. The following points may be considered:

- Molecular tests may be done in the first 24 hours of age.

- Repeat testing may be done at 48 hours of age.

- For well newborns who are likely to be discharged before 48 hours of age, clinicians may consider not doing the test.

- For each test, throat and nasopharyngeal swab must be taken and placed in the viral transport medium.

- For infants who remain in the hospital for on-going care, two tests obtained 24 hours apart which are negative should be considered for transitioning to universal precautions.

- For those infants who are positive in the initial PCR screening, subsequent follow-up testing should be done at intervals of every 48-72 hours until two tests are negative.

\section{What Samples can be Sent for TESTING ? ${ }^{10,11}$}

Sample collection is a very important preanalytical variable to ensure good results. Inappropriate sample collection can lead to false negative results which are likely to have implications on patient management and infection control precautions. ${ }^{11}$

Specimens can be collected from the upper respiratory tract and the lower respiratory tract as follows:

- Upper respiratory tract: all upper respiratory specimens must be collected using nylon, polyester, or Dacron flocked swabs on plastic shafts. Calcium alginate and cotton swabs with wooden shafts should not be used as they contain substances, which inactivate the virus and inhibit the PCR. ${ }^{11}$

- Nasopharyngeal swab (NP): insert the swab through the nares parallel to the palate (not upward) until resistance is encountered. The distance from the ear to the nostril of the patient can also be considered as indicating the distance to the nasopharynx. Gently rub and roll the swab. Leave swab in place for several seconds to absorb secretions. Slowly remove swab while rotating it.

- Oropharyngeal swab/throat (OP): ask the patient to open the mouth wide and say "Ahh" with the tongue sticking out. Then insert the swab into the posterior pharynx and tonsillar areas and rub it over both tonsillar pillars and posterior oropharynx and avoid touching the tongue, teeth, and gums.

- The NP and OP swabs should be placed in the same VTM tube to maximize the sensitivity of the test.

- A nasal midturbinate (NMT) swab also called deep nasal swab: tilt the patient's head back 70 degrees, and while gently rotating the swab, insert it less than one inch (about $2 \mathrm{~cm}$ ) into nostril (until resistance is met at turbinates). Rotate the swab several times against nasal wall and repeat in other nostril using the same swab.

- An anterior nares (nasal swab; NS): specimen is collected by inserting swab, at least $1 \mathrm{~cm}$ ( 0.5 inch) inside the nares and firmly sample the nasal membrane by rotating the swab and leaving it in place for 10-15 seconds. Sample both nares with same swab.

- Nasopharyngeal wash/aspirate or nasal aspirate (NA): specimen is collected by attaching a catheter to a suction apparatus. The patient is made to sit with the head tilted slightly backward. One milliliter to $1.5 \mathrm{~mL}$ of nonbacteriostatic saline $(\mathrm{pH} 7.0)$ is instilled into one nostril. The tubing is inserted into the nostril parallel to the palate (not upward). The catheter should reach the depth equal to distance from nostrils to outer opening of ear. Begin gentle suction/aspiration and remove catheter while rotating it gently. The specimen is then placed in a sterile viral transport media tube.

- Of the above, the NP and OP specimens are commonly used in clinical settings.

- Lower respiratory tract:

- Sputum-2-3 mL collected in a sterile leak proof screwcapped container for patients with productive cough. Induction of sputum is not recommended by Centers for Disease Control and Prevention (CDC, USA).

- Endotracheal secretions (ETS) - collected in a sterile leak proof screw-capped container from patients on invasive mechanical ventilation.

- Bronchoalveolar lavage (BAL) in a sterile leak proof container if clinically indicated in severely ill patients.

Whenever the lung or the lower respiratory tract is involved, it is always better to send sputum, ETS, or BAL as they are likely to have larger number of viral particles as compared to NP swabs.

\section{Precautions to be Taken When Collecting SAMPLES}

Specimen collection is an aerosol-generating procedure and the personnel collecting the sample must therefore wear appropriate personal protective equipment (PPE). The PPE should allow 
protection for contact, droplet, and airborne transmission and include gloves, disposable gown, N95 respirators or particulate filter respirators (FFP2 or FFP3), goggles or visors, and shoe covers. ${ }^{12,13}$ Visors may be more useful for pediatric sample collections. All HCWs working in this area should be appropriately trained for donning and doffing of PPE. In addition, training on swab or sample collection and ensuring appropriate forms are filled with necessary information for testing, storage of samples, and transport of sample should be done and also periodically reviewed..$^{12,13}$

\section{Specimen Storage}

All specimens can be stored at $2-8^{\circ} \mathrm{C}$ for up to 72 hours after collection. If a delay in testing or transporting is expected, store specimens at $-70^{\circ} \mathrm{C}$ or below. ${ }^{11}$

\section{Packaging}

All samples that need to be transported to a testing site away from the collection must ensure adequate biosafety precautions are taken to ensure prevention of spills and exposure. ${ }^{11} \mathrm{~A}$ triple layer packing with the outermost layer having a biohazard label impervious to fluids should be used. All samples placed inside should have their own containers and adequately protected with absorbent material for absorbing any spills.

\section{Transport ${ }^{11}$}

Transport of samples must be done using ice packs to maintain the temperature. For delayed specimen, transportation should be done using dry ice till it reaches the testing facility.

\section{What are the Methods Available for TESTING IN Laboratories?}

Tests for SARS-CoV-2 are based on two principles:

- Those that detect the viral proteins or the viral nucleic acid, i.e., ribonucleic acid (viral RNA) and give information on active infection and infectiousness of a person. These may be in the form of nucleic acid amplification tests (NAAT) or rapid antigen detection tests. This type of testing helps in control of the disease from spreading.

- Those that detect the body's response to the virus in the form of antibodies, IgM, and IgG indicating that the person is infected at some point in the past but not indicating infectiousness of the patient. This type of testing helps in understanding the extent of infection in the population.

Testing centers in India are authorized by Indian Council of Medical Research (ICMR) to perform testing for SARS-CoV-2. Laboratories must strictly adhere to biosafety precautions when processing clinical specimens as recommended by $\mathrm{WHO} .^{10}$ Tests available:

- Nucleic acid amplification tests (NAAT): NAATs or molecular methods are the most commonly used methods for detection of SARS-CoV-2. Unique genetic sequences of the viral RNA are detected with the help of NAAT methods like real time reverse transcription polymerase chain reaction (rRT-PCR) with confirmation by nucleic acid sequencing when necessary. ${ }^{10} \mathrm{The}$ World Health Organization (WHO) has identified two primer probe sets, which target the SARS-CoV-2 RNA-dependent RNA polymerase (rdRP) and envelope (E) genes for laboratory testing. ${ }^{10,14}$ The CDC assay contains primer probe sets for two regions of the viral nucleocapsid gene (N1 and $\mathrm{N} 2$ ) and also for the human RNase $P$ gene to ensure the RNA extraction was successful. The WHO and CDC assays have high analytic sensitivity and specificity with minimal cross-reactivity for other circulating strains of coronaviruses. A study by Corman et al. showed that the $R d R P$ and $E$ genes have high analytical sensitivity for detection with a technical limit of detection of 3.6 and 3.9 copies per reaction, respectively, whereas the $N$ gene has a poorer analytical sensitivity of 8.3 copies per reaction. ${ }^{15}$ The criterion for positivity in both assays is a cycle threshold ( $C_{\mathrm{t}}$ value) of less than $40 .^{3,16}$ RT-PCRs can be performed in one-step or a two-step assay formats. The one-step process provides rapid and reproducible results for high throughput analysis but generates lower target amplicons. In the two-step assay, both reactions are sequentially done in separate tubes, and while the assay is more sensitive, it is time-consuming and requires optimization of additional parameters. ${ }^{17,18}$ In RT-PCRs, it is important to select controls carefully to ensure reliability of the assay and identify experimental errors before interpretation is done. The sensitivity and specificity of RT-PCRs are not $100 \%$, and hence, a negative result in a specific epidemiological setting must be investigated with additional testing. Ren et al. found that when used alone, the RT-PCR has a sensitivity of $78.2 \%{ }^{19}$

As of 23/4/2020, ICMR has validated 37 real time PCR kits for use in India. ${ }^{20}$ ICMR recommends using the two-step protocol with the screening assay for the envelope protein (E gene) and a confirmatory assay using either the SARS-CoV-2 RNA-dependent RNA polymerase (rdRP) or the nucleocapsid protein gene (N gene). Many laboratories also use the single-step protocol. The real turn around time (TAT) for such assays is on an average 6-8 hours taking into consideration extraction, amplification, and detection. This may vary for different laboratories and can go up to 48 hours depending on availability of automation and man power resources from sample receipt to reporting.

Advantages of RT-PCRs:

- Large number of tests can be performed in batches with open real time PCR.

- Cost per test can be reduced as controls are used for a batch of samples.

Disadvantages: ${ }^{21,22}$

- Requires certified laboratories, dedicated infrastructure, expensive equipment, and trained personnel to perform the test.

- Long TAT of depending on volume of samples and automation available.

- An important limitation of PCR is that they do not differentiate between infective and noninfective viruses (dead or antibody neutralized).

\section{Automated Rapid Nucleic Acid Amplification Tests}

There are other NAATs also available which are low complexity rapid point of care devices based on cartridge or pouch formats where all the steps of the PCR process, right from extraction to detection are performed within one cartridge/pouch e.g. Chip based TrueNat (Molbio Diagnostics, India), Cartridge based Xpert Xpress SARS-CoV-2 cartridge (Cepheid, Sunnyvale, United States of America), isothermal nucleic acid amplification based Abbott ID NOW (Abbott Laboratories), real time nested pouch PCR based BioFire FilmArray (bioMérieux) and compact PCR based cobas Liat (Roche Diagnostics). ${ }^{23}$ More such rapid molecular platforms are under investigation and will be an important addition to the 
testing machinery as they are much simpler to handle and do not need expertise at the level on the laboratory. They are "sample to answer" formats which have a TAT of less than one hour. These closed PCRs play a very important role in rapid diagnosis as they can easily be placed as point of care devices (POC) in emergency departments and remote locations ensuring the presence of PPE, a Class 2 Biosafety cabinet and other biosafety considerations for safety of laboratory staff and clinical teams before initiating the process. The disadvantage of these rapid closed POC NAATs are that they may not be able to handle large volume of samples and the cost of these tests are much higher than the real time open PCRs. ${ }^{24}$

- Antibody testing methods: another type of test to detect infection with viruses like SARS-CoV-2 is by detecting the presence of antibodies, IgM, and IgG in the blood of infected patients. ${ }^{21}$ These are also called as serological methods. Antibodies used to develop these tests are virus neutralizing antibodies, those against the nucleocapsid proteins and the spike protein antigens including the $\mathrm{S} 1$ subunit protein. ${ }^{25}$ Antibody testing can be done using enzyme immunoassay (ELISA), chemiluminescence immunoassays (CLIA), or rapid lateral flow or chromatography assays (RDTs) which can be used as point of care tests. The TAT for these tests is about 30 minutes to 2 hours. Zhoa et al. studied 173 patients with COVID19 disease and found that the median time for seroconversion for IgM and IgG was days 12 and 14, respectively. In the first week of infection, antibodies were seen in $<40 \%$ of the patients which increased to $100 \%$ after day 15 of onset (IgM 94.3\%, IgG $79.8 \%) .{ }^{26}$ The strength of antibody formation depends on age, nutritional status, severity of disease, and infections like HIV and other conditions that suppress the immune system. ${ }^{21,26}$ The presence of antibodies was $<40 \%$ among patients within 1 week since onset and rapidly increased to $100.0 \%(\mathrm{Ab}), 94.3 \%(\operatorname{lgM})$, and $79.8 \%(\mathrm{lgG})$ since day 15 after onset. ${ }^{26}$ Most patients develop antibodies in the second week of onset of symptoms, which is usually the phase of recovery. At this stage, any opportunity for clinical intervention and interruption of disease transmission has already passed. ${ }^{21,26}$ Hence, antibody testing has little role to play in the active spread of the disease. Another important drawback to using antibody testing is that these antibodies may cross react with other human coronaviruses giving false positive results. ${ }^{25,27}$

\section{Utility of Antibody Testing 25,26}

- Since there is no previous exposure to SARS-CoV-2, presence of antibodies differentiates between infected and noninfected population.

- Antibody testing thus has a role in population surveillance to know how many got infected despite active surveillance measures.

- If molecular tests are negative and the serology comes positive, it indicates that the patient cannot transmit the infection which may have a role to play in transitioning patients from droplet to universal precautions and freeing isolation rooms, which are scarce in the current scenario.

- In critically ill patients who have been given plasma therapy or hyperimmune globulins, they are not likely to develop their own antibodies. This puts them at a risk of re-infection and this can be detected by antibody testing. These patients can also become prime candidates for a vaccine whenever available.

- Antibody testing is also critical in vaccine development to monitor the immune response to the vaccine.
- Zhoa et al. found that quantitative estimation of antibodies may help determine prognosis of the disease, as a study suggests that a higher antibody titer was associated with worse clinical outcomes $(<0.006)$.

\section{Current Status of Antibody Testing in India}

ICMR in its guidance dated 4th April, suggested the use of antibodies in conjunction with real time PCR. A series of kits have been validated by ICMR for the same. On 17th April 2020, ICMR issued a new advisory mentioning that rapid antibody kits should only be used as an epidemiological surveillance tool especially in designated hot spot zones in the community and not as a diagnostic tool. ${ }^{28}$ Similarly, WHO also does not recommend the use of antibody testing for patient care but encourages to study its usefulness for disease surveillance and epidemiologic research (WHO). ${ }^{29}$

- Antigen detection: another method of testing for SARS$\mathrm{CoV}-2$ is by detecting viral proteins expressed by the virus in a sample from the respiratory tract. This is called antigen detection and can be done as enzyme linked assays (ELISA) or chemiluminescent assays (CLIA) or in rapid lateral flow and chromatography formats as we use for dengue or for antibody testing. The TAT of such results would be about 30 minutes to 2 hours depending on what method is used. Since these antigens are expressed when the virus is actively replicating, such tests would be best to detect acute infection. However, the results depend on several factors like appropriate specimen collection, concentration of the virus in the specimen, and binding characteristics of the antigen to the antibody, etc. The sensitivity of such tests may vary from $34 \%$ to $80 \%$ based on previous experience with influenza. ${ }^{30}$ This leads to a large number of patients being missed and additional studies need to be conducted to understand its utility in the current pandemic. ${ }^{29}$ Another concern would be cross-reactivities with circulating coronaviruses causing false positives results. ${ }^{29}$ Still, these tests may have utility in triage and emergency departments to rapidly identify patients and reduce the need for expensive molecular tests. These assays are still in development for SARS-CoV-2. Due to lack of evidence, WHO has not recommended the use of such tests but recognizes the need for more research on their performance and clinical utility.

\section{Conclusion}

The COVID-19 pandemic has brought into focus the importance of microbiology laboratories in diagnosis and control of communicable diseases. There is an urgent need to scale up molecular testing based on available expertise and infrastructure and encourage healthcare facilities to undertake testing based on national guidance. Low complexity, rapid PCR assays may need to be scaled up for better patient management and infection control in remote and low volume settings. As the pandemic slows down, the need for serological methods will gain significance. There is also an urgent need to make long-term investments in scaling up reliable diagnostics with respect to COVID-19 disease.

\section{References}

1. WHO. Novel coronavirus-China. http://www.who.int/csr/don/12january-2020-novel-coronavirus-china/en/. Accessed April 23, 2020.

2. Zhu N, Zhang $D$, Wang $W$, et al. A novel coronavirus from patients with pneumonia in China, 2019. N Engl J Med 2020;382(8):727-733. DOI: 10.1056/NEJMoa2001017. 
3. Li Q, Guan X, Wu P, et al. Early transmission dynamics in Wuhan, China, of novel coronavirus-infected pneumonia. N Engl J Med 2020;382(13):1199-1207. DOI: 10.1056/NEJMoa2001316.

4. Siordia JA. Epidemiology and clinical features of COVID-19: a review of current literature. J Clin Virol 2020;127:104357. DOI: 10.1016/j. jcv.2020.104357.

5. https://www.icmr.gov.in/pdf/covid/strategy/Testing_Strategy_ v5_18052020.pdf.

6. https://services.aap.org/en/pages/2019-novel-coronavirus-covid-19infections/faqs-management-of-infants-born-to-covid-19-mothers/.

7. https://www.aappublications.org/news/2020/04/02/ infantcovidguidance 040220.

8. He X, Lau EH, Wu P, et al. Temporal dynamics in viral shedding and transmissibility of COVID-19. Nat Med 2020;26(5):672-675. DOI: 10.1038/s41591-020-0869-5.

9. Wölfel R, Corman VM, Guggemos W, et al. Virological assessment of hospitalized patients with COVID-2019. Nature 2020. DOI: 10.1038/ s41586-020-2196-x.

10. World Health Organization (WHO). Laboratory testing for coronavirus disease 2019 (COVID-19) in suspected human cases. Interim Guid. 2020;(March):1-7.

11. https://www.cdc.gov/coronavirus/2019-ncov/lab/guidelines-clinicalspecimens.html.

12. World Health Organization (WHO). Infection prevention and control of epidemic- and pandemic-prone acute respiratory infections in health care. WHO Guidel. 2014;1-156.

13. European Center for Disease Control (ECDC). Infection prevention and control for COVID-19 in healthcare settings-first update 12 March 2020.

14. Corman VM, Landt O, Kaiser M, et al. Detection of 2019 novel coronavirus (2019-nCoV) by real-time RT-PCR. Euro Surveill 2020;25(3):2000045. DOI: 10.2807/1560-7917.ES.2020.25.3. 2000045.

15. Corman V, Bleicker T, Brünink S, et al. Diagnostic Detection of Wuhan Coronavirus 2019 by Real-Time RT-PCR. Geneva: World Health Organization; 2020.

16. Cheng MP, Papenburg J, Desjardins $M$, et al. Diagnostic testing for severe acute respiratory syndrome-related coronavirus-2: a narrative review. Ann Intern Med 2020. M20-1301. DOI: 10.7326/ M20-1301.
17. Wong ML, Medrano JF. Real-time PCR for mRNA quantitation. BioTechniques 2005;39(1):75-85. DOI: 10.2144/05391RV01.

18. Pfaffl MW. Quantification strategies in real-time PCR. In: Bustin SA A-Z of quantitative PCR. La Jolla, San Diago CA, USA: International University Line (IUL; 2004. pp. 87-112.

19. Jiang G, Ren X, Liu Y, et al. Application and optimization of RT-PCR in diagnosis of SARS-CoV-2 infection. medRxiv 2020.

20. https://icmr.nic.in/sites/default/files/upload_documents/Real_time_ PCR_tests_23042020.pdf.

21. Li Z, Yi Y, Luo X, et al. Development and clinical application of a rapid IgM-Ig G combined antibody test for SARS-CoV-2 infection diagnosis. J Med Virol 2020. DOI: 10.1002/jmv.25727.

22. Joynt GM, Wu WK. Comment understanding COVID-19: what does viral RNA load really mean? Lancet Infect Dis 2020. DOI: 10.1016/ S1473-3099(20)30237-1.

23. Tang Y-W, Schmitz JE, Persing DH, et al. The laboratory diagnosis of COVID-19 infection: current ssues and challenges. J Clin Microbiol 2020;4:JCM.00512-JCM.00520.

24. World Health Organization (WHO). Rapid communication on the role of the GeneXpert ${ }^{\oplus}$ platform for rapid molecular testing for SARSCoV-2 in the WHO European Region European Laboratory Initiative on TB, HIV and Viral Hepatitis. Copenhagen;1st April 2020.

25. Okba NMA, Müller MA, Li W, et al. Severe acute respiratory syndrome coronavirus 2-specific antibody responses in coronavirus disease 2019 patients. Emerg Infect Dis 2020;26(7). DOI: 10.3201/eid2607.200841.

26. Zhao J, Yuan $\mathrm{Q}$, Wang $\mathrm{H}$, et al. Antibody responses to SARS-CoV-2 in patients of novel coronavirus disease 2019. SSRN Electron J 2020. ciaa344. DOI: 10.1093/cid/ciaa344.

27. Che X, Qiu L, Liao Z, et al. Antigenic cross-reactivity between severe acute respiratory syndrome-associated coronavirus and human coronaviruses 229E and OC43. J Infect Dis 2005;191(12):2033-2037. DOI: $10.1086 / 430355$.

28. https://icmr.nic.in/sites/default/files/upload_documents/Rapid_ Antibody_test_Protocol.pdf.

29. https://www.who.int/news-room/commentaries/detail/advice-onthe-use-of-point-of-care-immunodiagnostic-tests-for-covid-19.

30. Bruning AHL, Leeflang MMG, Vos JMBW, et al. Rapid tests for influenza, respiratory syncytial virus, and other respiratory viruses: a systematic review and meta-analysis. Clin Infect Dis 2017;65(6):1026-1032. DOI: 10.1093/cid/cix461. 